\title{
The GRIPP checklist: Strengthening the quality of patient and public involvement reporting in research
}

\author{
Sophie Staniszewska, Jo Brett, Carole Mockford \\ University of Warwick \\ Rosemary Barber \\ University of Sheffield
}

Objectives: The aim of this study was to develop the GRIPP (Guidance for Reporting Involvement of Patients and Public) checklist to enhance the quality of PPI reporting. Methods: Thematic analysis was used to synthesize key issues relating to patient and public involvement (PPI) identified in the PIRICOM and PAPIRIS systematic reviews. These issues informed the development of the GRIPP checklist.

Results: The key issues identified included limited conceptualization of PPI, poor quality of methods reporting, unclear content validity of studies, poor reporting of context and process, enormous variability in the way impact is reported, little formal evaluation of the quality of involvement, limited focus on negative impacts, and little robust measurement of impact. The GRIPP checklist addresses these key issues.

Conclusion: The reporting of patient and public involvement in health research needs significant enhancement. The GRIPP checklist represents the first international attempt to enhance the quality of PPI reporting. Better reporting will strengthen the PPI

evidence-base and so enable more effective evaluation of what PPI works, for whom, in what circumstances and why.

Keywords: Patient and public involvement (PPI), Impact, Checklist, Reporting, Quality

Patient and public involvement (PPI) in Health Technology Assessment (HTA) and health research has become increasingly common internationally. Interest and activity have grown, with projects in the European Union, Australia, Canada, and other countries focusing on different aspects of PPI $(1 ; 3 ; 6 ; 11 ; 14 ; 18 ; 20 ; 21 ; 24)$. There is a general agreement on the need for more patient-focused HTA methods

We would like to acknowledge the contribution of the research teams and advising groups for PIRICOM and PAPIRIS to these two original reviews. The PIRICOM systematic review was funded by the United Kingdom Clinical Research Collaboration. The PAPIRIS systematic review was funded by the National Centre for Involvement, UK. and several HTA agencies and HTA researchers are reviewing ways to incorporate the patients' or, more generally, the public's perspectives into their methods (9). However, the need for evidence through robust evaluation has also been emphasized to convince a broader constituency of the HTA community about the impacts of PPI $(10 ; 22)$. In the United Kingdom, the Director General of NHS Research and Chief Medical Officer has recently stated that involvement should be the norm, not the exception, in health research (including HTA), although progress is still needed to implement this vision. The overall aims of involvement are to enhance the quality, relevance, and appropriateness of research and 
also to contribute to the broader democratization of research, through participatory forms of involvement that encourage partnership in research $(4,6)$. Within the United Kingdom, considerable effort has been focused on developing an infrastructure to operationalize the policy commitment to PPI, through the work of organizations such as INVOLVE (12) and the Research Design Services (19), which enable researchers to embed patient and public involvement into their work. Together, the policy focus and the infrastructure in the United Kingdom and in other countries have created a supportive context in which PPI activity has flourished.

While PPI activity has continued to expand, there has been relatively little scrutiny of the difference it makes. We undertook two recent systematic reviews, PIRICOM (4) (Patient and Public Involvement in Research: Impact, Conceptualisation, Outcomes and Measurements) which examined the impact of patient and public involvement on health and social care research, and PAPIRIS (15) (Patient And Public Involvement Review on the Impact on healthcare Services), which focused on the impact of patient and public involvement on health service provision, evaluation, and delivery. Both reviews examined the conceptualization, definition, measurement, impact, and outcomes of patient and public involvement in their respective areas. The full results of each review are reported elsewhere $(4 ; 15)$. INVOLVE have also recently conducted a structured review of the impact of user involvement in research (21).

While the results of PIRICOM (4), PAPIRIS (15), and the INVOLVE study (21) have been helpful in identifying a range of impacts that were attributed to patient and public involvement, it has become clear that the underlying evidence base is relatively poor, primarily because of poor reporting, making it difficult to assess the impact of involvement. Problems with reporting in health and social care research are not unusual, and so difficulties in reporting patient and public involvement research are not exceptional. In health research, there is evidence that many papers lack clarity, transparency, and completeness in how the authors actually carried out the research $(2 ; 16 ; 17)$. Poor reporting can cause a range of difficulties. If readers do not have sufficient details concerning a study, they are left with an incomplete picture which makes its appraisal very difficult and has implications for the judgment of reliability and the interpretation of results $(16 ; 17)$. In addition, there are moral and ethical imperatives for the good reporting of research which cannot be ignored. The lack of adequate reporting in studies prompted the creation of the EQUATOR Network, which is an international initiative that seeks to enhance the reliability and value of medical research by promoting transparent and accurate reporting of research studies $(7 ; 13)$. EQUATOR recognized that randomized controlled trials were often reported poorly, which fuelled the development of the original CONSORT (CONsolidated Standards of Reporting Trials) statement in 1996 with its revision 5 years later (17), and an updated recently published version, CONSORT 2010 (16). The most recent statement aims to assist authors in writing reports of randomized controlled trials, editors and peer reviewers in reviewing manuscripts for publication, and readers in critically appraising published articles. While the EQUATOR network has been very helpful in enhancing the quality of research reporting, there is little similar guidance for researchers reporting patient and public involvement, beyond more general guidance for reporting the different types of qualitative and quantitative research and recently published guidelines for appraising the quality and impact of user involvement in research, which relies on good quality reporting (26). This paper addresses this deficit by reporting the findings of a follow-up study, which synthesized the key issues from the PIRICOM and PAPIRIS systematic reviews and proposed the GRIPP checklist (Guidance for Reporting Involvement of Patients and Public), to enhance the quality of PPI reporting in HTA and in health research more broadly to enable quality assessment. The GRIPP checklist is the first attempt to comprehensively identify the important elements of good reporting for PPI, based on systematic reviews of the PPI evidence base. The aim of the GRIPP checklist is to help researchers and service users improve the quality, content, detail, consistency, transparency, and completeness of their PPI reporting, thus strengthening the PPI evidence base, and ultimately enabling a clearer understanding of what PPI works, for whom, why and in what circumstances.

The aim of this study was to develop the rationale and content of the GRIPP checklist.

\section{METHODS}

The PIRICOM (http://www.ukcrc.org/publications/reports/) and PAPIRIS studies both used the York Centre for Reviews and Dissemination Guidance (5) for undertaking systematic reviews. The PIRICOM study recruited three service users to the advisory board, who were involved in developing the study aims, analysis, interpretation, writing, and dissemination. An expert seminar with twenty-four service users and researchers in the field was held to assist with the interpretation of the evidence base. The full findings are reported elsewhere (4). The PAPIRIS study was also guided by an advisory group, consisting of twelve leading experts in patient and public involvement and systematic reviews, including two lay members. The advisory group assisted at all key stages specifically the protocol stage, data retrieval, and results stages. The full findings are reported elsewhere (15).

As the PIRICOM and PAPIRIS reviews progressed several issues relating to poor quality of reporting were identified. A separate study, following on from PIRICOM and PAPIRIS, was undertaken, in which narrative synthesis of each key issue was undertaken, comparing their type and nature across both reviews, to enable an interrogation by S.S., J.B., C.M., and R.B. of each issue. The following section describes these key issues, and provides the underpinning rationale for the GRIPP checklist presented in Table 1. While 


\section{Abstract:}

1a. Aims: The specific aim relating to PPI or capturing or measuring impact of PPI must be included

1b. Results: State that PPI has been included and that impact has been assessed. Report any key impacts.

1c. Keywords: Include 'PPI' or a derivation, and 'impact' as keywords.

\section{Background/literature review}

2a. Definition: Provide a definition of PPI and ideally link this to definitions provided by other studies, providing a rationale for that definition. For example, the definitions used by any other studies that have developed methods or instruments to measure impact.

2b. Conceptualisation and theoretical development: Provide a clear account of the way in which PPI is being conceptualised and whether the study is drawing on any conceptual or theoretical underpinnings, including any conceptual theoretical models or influences.

3. Aims: Include the aim of PPI capture or measuring impact of PPI.

4. Methods: Report the detail of the PPI activity, in terms of a

4a. Clear description of all involvement activity,

4b. Description of participants, both users and researchers,

4c. What level of PPI was utilised (consultation, collaboration, user-led),

4d. Whether PPI occurred at one stage or multiple stages of research,

4e. A detailed description of PPI at each of the relevant research stages, for example, developing aims, selecting methods, data collection, data analysis, writing and dissemination,

4f. The research design that was used e.g. focus group, interviews, and diaries.

5. Capture of PPI Impact: Report the method used to capture impact, for example, when qualitative, describe this process.

6. Measurement: Report the method used for how any quantitative assessment of impact has been made and on the robustness of this assessment.

7. Economic appraisal: Report the method used for any economic assessment of PPI impact.

8. Analysis:

8a. Report how users have been involved and

8b. Report how users influenced the analysis, interpretation and synthesis of impact data.

\section{Results:}

9a. Context: Report results in a broader framework that considers the contextual factors underpinning the study. Comment on the way any contextual factors have enabled, hindered or otherwise influenced PPI activity, impact and outcomes.

9b. Process: Report results in a broader framework which considers process factors that may have affected impacts.

9c. Conceptualisation/theoretical development: Report any comments on conceptualisation of PPI, as operationalized in this study and any key messages for future studies, particularly those who wish to utilise conceptual or theoretical models to develop instruments to measure impact. Comment on how well any original conceptual model match the dimensions of impact identified by the study.

9d. Testing of conceptual or theoretical models: This needs to be reported in extensive detail as there is so little testing.

9e. Impacts and outcomes: Report all aspects of impact and outcomes, both important impacts and more minor ones. Report both positive and negative impacts and also evidence of no impact, and consider the possibility that positive or negative impacts may be in the eye of the beholder and so interpretation will vary. If capture of impact is qualitative include adequate detail of the impact, its nature and any influences from or relationships with context and process factors.

9f. Measurement: If an instrument or method to measure PPI impact was developed or utilised, report all aspects of instrument development and testing, including how users influenced it, how well the instrument performed, justification of content and face validity, any data on reliability and other forms of validity.

9g. Economic appraisal: Report any information on the economic cost or benefit of PPI.

\section{Discussion and conclusions:}

10a. Definition: Comment on how effectively the definition of PPI adopted in this study has been operationalized, and make any suggestions for how future studies should define PPI.

10b. Conceptualisation/theoretical development: Report how the findings contribute to a broader theoretical thinking, how their study builds on others and how future studies could utilise the conceptual information contained in this study.

10c. Context and process: Comment on the importance of context and process factors and any relationship with any aspects of impact.

10d. Impact and outcomes: Comment on the nature, content and extent of impact, and how impacts identified in this study contribute to the broader knowledge base of impact, and the relationship between specific impacts and specific context and process factors, clearly justifying this. Assess the extent to which content validity has been achieved.

10e. Measurement: Comment on how adequately impact has been measured and any key limitations. Make any suggestions for future instrument development.

10f. Economic information: Discuss any information on the economic cost or benefit of PPI, particularly any suggestions for future economic modelling.

10g. Critical perspective: Comment critically on the study, reflecting on the things that went well and those that did not, so others can learn from this experience.

Note. This checklist is designed for studies that have included some form of patient and public involvement in research. The aim of the GRIPP checklist is to assist authors in writing their PPI papers and reports, editors and peer reviewers in reviewing manuscripts for publication, and readers in critically appraising published articles and reports. 
the key issues are concerned with reporting, some have implications for how the future PPI evidence base PPI should evolve, particularly in relation to the way in which PPI impact should be evaluated and measured (22). These implications are briefly discussed in the paper.

\section{RESULTS}

\section{Conceptualizing or Theorizing PPI}

Good reporting is ideally based on clear concepts and definitions. While there are some helpful definitions of involvement, the conceptualization or theorization of PPI has generally been poor. There have been some attempts to develop conceptual or theoretical frameworks, but there is no overall conceptual model of PPI impact that captures the essence of the concept and has been empirically tested. Such models can be very helpful because they can provide a blueprint for evaluation, identifying key areas for assessment. The often poor reporting of definitions and the lack of an agreed conceptual framework or model for PPI was problematical in assessing the conceptual equivalence of studies, that is, the extent to which they were examining the same concept $(4 ; 15)$. The lack of conceptual frameworks or models also made it difficult when attempting to assess impact of PPI. Both the PIRICOM and PAPIRIS reviews adopted a broad approach and regarded impact as the reporting of any information concerned with the difference patient and public involvement has made to any aspects of research, researchers, patients and the public, users, organizations, or to service delivery, development, or evaluation, to ensure all potentially relevant information was included. The lack of a clear conceptual or theoretical underpinning for the reporting of impact may explain why studies have adopted a variety of approaches, epitomizing the phrase "letting a thousand flowers bloom." While this provides a fascinating insight into the richness and diversity of patient and public involvement, it hinders consistent and more formal evaluation of impact.

\section{Reporting of Methods}

Studies of patient and public involvement varied enormously in the data provided about the PPI activity, the level of PPI adopted (consultative, collaborative, or user-led), the stages at which the activity occurred (whether one stage or multiple stages), and the research design used. A key difficulty was that methods were often described in very little detail and often inconsistently with varying information. It was rare to have a full description of participants, both service users and researchers. In addition, authors rarely identified the level or levels of involvement (consultation, collaboration or userled) they used, leaving it to the reader to form an assessment. The stages at which PPI occurred in a study were also poorly reported, and it was often unclear whether PPI had occurred at one point or throughout a study.

\section{Content Validity of Studies}

A key difficulty with many studies of PPI was that they rarely assess the content validity of their investigation. Content validity is an assessment of the extent to which a study or an instrument captures all the relevant dimensions or aspects of a concept (23). Ideally the assessment of content validity would be based on some conceptual or theoretical understanding of a concept. For example, when Ware and Sherbourne (25) developed the Short-Form 36 (SF-36) questionnaire they stated that health comprised eight concepts including physical function, role physical, bodily pain, mental health, role emotional, social function, general health, and vitality. Thus, to ensure content validity, any generic patient reported outcome measure (PROM) should measure all eight concepts. This conceptual framework provides guidance on which dimensions of health all generic patient reported outcome measures should include, and offers a basis for the assessment of content validity. While content validity is a standard part of evaluating PROMs, the concept could also be transferred to studies of patient and public involvement. PPI studies rarely report the extent to which they have considered all the potentially relevant dimensions or aspects of PPI, particularly important when evaluating impact. Using the concept of content validity in the field of PPI could enable more consistent reporting of all relevant aspects of a study and so enhance future evaluation of the full spectrum of possible impacts.

\section{Reporting of Context and Process}

The PIRICOM review, in particular, through collaboration with patients and the public, identified the importance of context and process in the interpretation of PPI impact (4). Context refers to the conditions required for PPI to have an impact. For example, the appropriate support and training, the appropriate funding, positive attitudes toward PPI, and appropriate time allocation might be important in a particular situation. Process refers to the methods used to undertake the involvement (see Figure 1), such as level of involvement and the stages of the research process where involvement occurs. While some studies did describe context and process information, although in varying detail, it was rarely linked to any interpretation of impact. The importance of context and process suggests that PPI should be viewed as a complex intervention that requires multi-layered reporting.

\section{The Status of Impact}

While PIRICOM and PAPIRIS identified a wide range of studies that reported on various PPI activities, relatively few papers included an evaluation of the impact of involvement as a primary aim, possibly because studies focused on assessing the effectiveness of an intervention. This may indicate the low status accorded to PPI and the difference it makes and may explain why less emphasis has been placed on consistent reporting. 


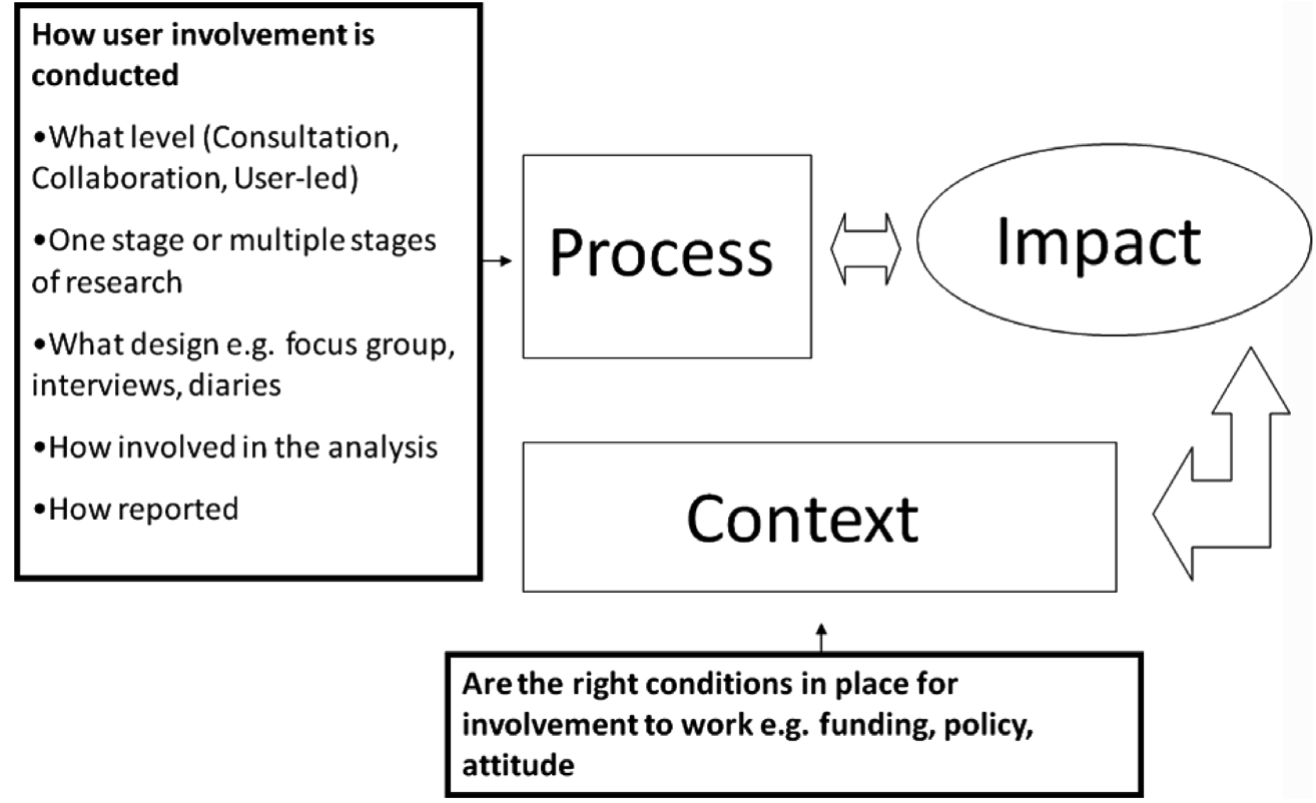

Figure 1. The complexity of PPI impact evaluation.

\section{Locating the Evidence of Impact}

In searching for and selecting papers for the PIRICOM and PAPIRIS review, it became apparent that there were some significant challenges in developing a sensitive and specific search strategy, which identified relevant papers, while excluding irrelevant ones. A key difficulty was the lack of MeSH (Medical Subject Headings) for PPI, which if they existed, could offer an efficient way of finding relevant literature. Instead authors have used a wide variety of keywords, often in an inconsistent way, which limited the utility of keyword searching and made the development of a search string very complex. A further difficulty related to using the title and abstract information as a way of identifying relevant studies, a common approach in reviewing and helpful in sorting through large numbers of studies. Sifting PPI studies by title and abstract was not a useful approach as information about impact was not always mentioned in the title or abstract, but could appear in another part of the paper, such as the results or discussion. As a result, entire papers had to be read for a robust assessment of relevance, which had a significant impact on the time needed for the review of papers. Such difficulties have important implications for future syntheses of evidence as the same difficulties will remain unless there is more emphasis placed on appropriate reporting in the title or abstract and appropriate use of keywords.

\section{Locating Impact Information in a Paper}

In addition to difficulties locating and selecting relevant papers, the PIRICOM and PAPIRIS studies also highlighted important difficulties locating information about impact within papers. Information about impact could appear anywhere in a paper and there was no consistent place where authors reported impact, and which a reviewer could consistently use during data extraction. Rarely was there a full and consistent appraisal of impact data with information about impact appearing in all the appropriate sections of a paper, (for example as an aim, in the methods section, or in the results, discussion, and conclusion) to allow a full appraisal of the difference PPI made in the study. More commonly, information about impact appeared as a one-off comment, which could occur anywhere in the paper. This inconsistency in reporting meant that papers had to be read in full for the purposes of data extraction, a very inefficient way of identifying and extracting relevant information which normally relies on key data appearing in expected parts of the paper.

In addition to the difficulties of finding the evidence, there was a range of issues that inhibited the appraisal of information about impact which relates to the nature of the PPI evidence base more broadly, including the poor conceptualization and theorization of impact. These issues need to be addressed as part of strengthening this evidence base alongside the development of better reporting and are briefly considered in the conclusion.

\section{The Level of Detail About Impact}

Both the PIRICOM and PAPIRIS reviews attempted to extract data on impact or the difference PPI made to research or to a health service. Any form of data was sought, including qualitative descriptions or quantitative measurements. Of the studies that considered impact, most reported impact using short descriptions. These were often very limited, lacking in detail and with no consistent structure. Descriptions of 
impact usually took the form of one or two lines of text and were not always described as a PPI impact, meaning that the researcher had to decide that this data represented impact. During data extraction, the identification of longer sections of text reporting impact were like "nuggets of gold." In the absence of formal and structured forms of reporting, the most helpful types of report in the PAPIRIS study were the small number of case studies, often contained in longer reports, which were time-consuming to read and not always peer-reviewed.

While helpful in providing some insight and enabling the identification of broad groupings of impact (impact on research, researchers, service users, community, policy, funders, journals), overall the level of reporting was inadequate if the reader's aim was to understand properly the difference that patient and public involvement had made, to use this information to develop a more coherent conceptual or theoretical model of impact, or to use such information to develop a bank of items for the development of an instrument that includes all relevant dimensions of impact. In many ways, the nature of current patient and public involvement reporting could be compared with a scenario where a researcher reports the findings of a randomized controlled trial (RCT) by stating that "a particular intervention has worked and patients feel better," and there is no further information. Such poor reporting would obviously impede a rigorous assessment of the effectiveness of an intervention. Such difficulties in RCT reporting prompted the development of CONSORT guidance to help improve the quality of reporting $(16 ; 17)$.

\section{Positive and Negative Impacts}

In reporting the impact of PPI most studies report positive impacts, with negative impacts more rarely considered. This may reflect an implicit assumption that PPI is a good and worthwhile activity, or that negative impacts have been politically more difficult to report. In ensuring content validity, papers should report both positive and negative impacts and also evidence of no impact, to enable a comprehensive understanding of the full breadth of PPI impact in a particular study. The need for such a comprehensive approach to reporting positive and negative impacts is particularly important for the development of robust instruments to measure impact.

\section{Quality of PPI}

Current approaches to assessing the quality of research focus on evaluating the study design but do not usually include PPI as an element of quality. There was no formal way of evaluating the quality of PPI or the quality of PPI impact reporting when the PIRICOM and PAPIRIS studies were conducted. In many respects, this might reflect the lack of critical evaluation which pervades much of the PPI evidencebase. More recently, guidance on evaluating the quality of PPI has emerged and may help strengthen quality assessment in this area (26). This guidance aims to help reader's assess the quality of published studies, researchers to develop effective strategies for user engagement, and funding bodies to establish principles of effective user involvement. It relies on good quality reporting, which the GRIPP checklist will seek to encourage.

\section{Capture and Measurement of PPI}

The main ways in which PPI impact is represented is through short descriptions. No standard formats exist for describing or capturing these impacts, and so they tend to vary in content, structure, and presentation. While other areas, such as patient experiences or patient-reported outcome measures have developed instruments which, with varying degrees of success, measure the concept of interest, PPI does not have a pool of robust, well developed instruments to measure PPI impact (25). Robust measurement of the extent of PPI impact could provide additional information that could enable a greater understanding of what works, for whom and in what circumstances. The application of psychometrically derived methods of measurement has much to offer PPI in developing robust instruments to measure the impact, context, and process.

\section{Economic Aspects}

In exploring the impacts of patient and public involvement, it is important to consider all potential impacts, including economic impacts, particularly if forming a judgment about whether a particular involvement activity is cost-effective. In the PIRICOM and in the PAPIRIS systematic reviews, there was no evidence of any economic modeling of costs or benefits, with only a very small number of papers mentioning costs of particular patient and public involvement activities. It is important that, in future theorizing of involvement, economic impacts are considered alongside forms of impact as part of a broader development of the patient and public involvement evidence-base.

\section{A Linked Body of Research}

The PIRICOM (4) and PAPIRIS (15) systematic reviews both identified a large number of diverse studies that have examined different aspects of patient and public involvement. However, this diversity poses several difficulties when attempting to synthesize different types of information about patient and public involvement including impact. Many studies simply report the results of a project, not always linking their study to a much broader body of work, or clearly outlining how their work moves thinking forward conceptually or methodologically. We risk reinventing the wheel many more times unless greater efforts are placed on developing a coherently linked area of work. This provides significant challenges for researchers in stating explicitly how their study adds to the body of knowledge to enable the conceptual and methodological developments to be clearly visible. 


\section{Reflective Evaluation and Interpretation}

The ability to critique and to reflectively evaluate and interpret research underpins the development of conceptual and methodological thinking. While such reflective thinking is present in the field of PPI, it tends to be found in opinion or reflective articles and less often in studies reporting the results of a project trying to establish the impact of PPI. For example, when studies presented their findings in relation to impact, the short descriptions (which formed the main way in which such data were captured) were rarely accompanied by any critical reflection. There was rarely any attempt to interpret or explain any relationship with context and process in evaluating impact. As a result, there is an important need to develop this critical reflective capacity in the reporting of impact, to enable the reader to critically assess a study and judge its merit and contribution to our broader understanding of patient and public involvement.

\section{Development of the GRIPP Checklist}

This paper has reported the narrative synthesis of the key issues that emerged from the PIRICOM and PAPIRIS systematic reviews and were primarily concerned with the poor quality of reporting within PPI studies. To develop the GRIPP checklist in this follow-up study, the research team carefully considered each issue in relation to several criteria: (i) whether the information was important to report within a paper that included some level of PPI, (ii) whether it would contribute to enhancing the evidence-base of PPI reporting more generally, and (iii) where the information should be reported to create greater transparency and so enhance the ease of future synthesis.

Criteria iii was useful for considering where information about an aspect of PPI should be reported within the structure of a paper, to enhance the quality of reporting. These deliberations helped to structure the checklist according to the key sections usually expected within a paper. The aim was to create a checklist that was logically structured and could be easily used by authors in writing their PPI papers and reports, editors and peer reviewers in reviewing manuscripts for publication, and readers in critically appraising published articles and reports.

\section{DISCUSSION}

This follow-up study has presented a synthesis of the key issues from the PIRICOM (4) and PAPIRIS (15) systematic reviews in relation to the reporting of patient and public involvement. If we are to understand the aims, methods, processes, and impact of PPI in HTA and health research more generally, it is important that there is a significant improvement in the quality of PPI reporting. This applies to a range of outputs, including peer-reviewed papers, case studies, and HTA reports. There have been recent suggestions by Facey et al. (8) that all HTA reports include a section on patient issues.
At present, the PPI evidence base is like an ice-berg, only partly visible within the literature, with much information hidden, either not reported or poorly reported. However, the absence of this information does not mean absence of activity or impact within individual studies. The GRIPP checklist represents the first international attempt to develop a checklist that provides robust guidance to enhance the quality of PPI reporting. It can be used by researchers, reviewers, editors, service users, and policy makers with any paper that attempted to include PPI, particularly those that have attempted to evaluate the impact of PPI. The GRIPP checklist has been registered with EQUATOR (http://www.equatornetwork.org/) and a process of consensus development will start shortly, in collaboration with EQUATOR and with other key international stakeholders. This process will develop international consensus and further refine and test the GRIPP checklist to ensure its robustness.

The GRIPP checklist is relevant for studies that are primarily reporting the results of their PPI activity. For other studies where the PPI component may be a secondary aim, for example, clinical studies that have evaluated an intervention and have included PPI, there is a need for authors to consider the utility of the checklist items for their own work. Ideally, authors should be encouraged to publish a separate PPI methods paper produced utilizing the GRIPP checklist, as this would provide a significant contribution to the broader evidence base. However, we recognize this is not always possible and we hope to provide firmer guidance in the future on the GRIPP checklist items that are vital, as opposed to optional, for studies where PPI is a secondary or tertiary aim. This deliberation will form part of the next phase of consensus development with EQUATOR and other stakeholders.

\section{Strengths and Weaknesses}

While the GRIPP checklist presented in this paper is based on two systematic reviews undertaken using robust methods, it has some limitations. A key limitation is the lack of international input to date. While PIRICOM included international studies. PAPIRIS only focused on UK-based studies, as it was concerned with health and social care services in the UK. The next stage of this work will include international collaboration to ensure a broad view is taken in developing consensus and in ensuring the robustness of the GRIPP checklist. However, as PIRICOM included international studies, we predict the GRIPP checklist has international relevance, although this will require further testing.

\section{Implications for Policy Makers}

The development of a stronger PPI evidence base, through better quality reporting, will enable policy makers to form a clearer understanding of the impact or difference that PPI makes to research. Such evidence may be vital in times of fiscal constraint where philosophical arguments that support PPI based on societal good are harder to defend. The 
strengthened PPI evidence base will also provide policy makers with an important recognition of the validity and relevance of PPI as an activity that strengthens the quality of research and so ultimately underpins evidence-based policy making.

\section{CONCLUSIONS}

The GRIPP checklist represents the first attempt to provide guidance that can be used internationally, to enhance the quality of PPI reporting to strengthen the future PPI evidence base. This will enable a wider range of audiences, including researchers, service users, and policy makers to better understand the impact, or difference, PPI can make to research. Elements of better reporting depend on future developments in the PPI evidence base. For example, it is important for future researchers to consider the importance of context and process in the interpretation and reporting of impact. This has obvious implications for the design and data collection stages of a study. Another key area where the PPI evidence base needs enhancement, to enable better reporting, is the development of robust instruments to quantitatively measure the impact of patient and public involvement (22). This will enable researchers, policy makers, and others to form better judgments about where PPI has greatest impact, the extent of the impact, and its nature. There is also a need for greater clarity in the conceptualization, definition, and theorization of PPI to ensure studies have conceptual equivalence, and so are comparing the same concept. Such changes require a paradigm change in PPI, moving it from an area that relies on case studies and narrative data to one that builds on this data by embracing more quantitative forms of evidence with known properties of reliability and validity that demonstrate the extent of impact more effectively. This requires a change in the nature of studies undertaken, and a need for funders to support the development of robust quantitative instruments that measure impact. Such changes in the nature of the PPI evidence base, together with better quality reporting, facilitated by the GRIPP checklist, will reveal the hidden iceberg of PPI evidence, thus enabling more effective future evaluation of what PPI works, for whom, why and in what circumstances.

\section{CONTACT INFORMATION}

Sophie Staniszewska, DPhil (Oxon) (sophie. staniszewska@warwick.ac.uk), Jo Brett, MSc, MA (J.Brett@warwick.ac.uk), Carole Mockford, DPhil (Oxon) (C.Mockford@warwick.ac.uk), Royal College of Nursing Research Institute, School of Health and Social Studies, University of Warwick, CV4 7AL Warwick, UK

Rosemary Barber, MSc (rosemary.barber@ sheffield.ac.uk), University of Sheffield, School of Health and Related Research, Regent Coord, 30 Regent Street, Sheffield S1 4DA, UK

\section{CONFLICT OF INTEREST}

All authors report they have no potential conflicts of interest.

\section{REFERENCES}

1. Abma T. Patient participation in health research: Research with and for people with spinal cord injuries. Qual Health Res. 2005; 15:1310-1328.

2. Begg C, Cho M, Eastwood S, et al. Improving the quality of reporting of randomized controlled trials. The CONSORT statement. JAMA. 1996;276:637-639.

3. Boote J, Telford R, Cooper C. Consumer involvement in health research: A review and research agenda. Health Policy. 2002;61:213-236.

4. Brett J, Staniszewska S, Mockford C, et al. The PIRICOM Study: A systematic review of the conceptualisation, measurement, impact and outcomes of patient and public involvement in health and social care research. London: United Kingdom Clinical Research Collaboration; 2009.

5. Centre for Reviews and Dissemination. Systematic reviews: CRD's guidance for undertaking reviews in health care. York: CRD, University of York; 2009.

6. Coupland H, Maher L, Enriquez J, et al. Clients or colleagues? Reflections on the process of participatory action research with young injecting drug users. Int J Drug Policy. 2005;16:191-198.

7. EQUATOR Network. https://www. equator-network.org/home/ (accessed Feb 11, 2011)

8. Facey K, Boivin A, Gracia J, Hansen HP, Lo Scalzo A, et al. Patients' perspectives in health technology assessment: A route to robust evidence and fair deliberation. Int J Technol Assess Health Care. 2010;26:334-340.

9. Gagnon M, Desmartis M, Lepage-Savary D, et al. Introducing patients' and the public's perspectives to health technology assessment: A systematic review of international experiences. Int J Technol Assess Health Care. 2011;27:31-42.

10. Gauvin F, Abelson J, Giacomini M, et al. Moving cautiously: Public involvement and the health technology assessment community. Int J Technol Assess Health Care. 2011;27:43-49.

11. Health Canada [WWW document]. URL http://www.hcsc.gc.ca/ahc-asc/serviceuser-consult/res-centre/poli-eng.php (accessed February 2011).

12. INVOLVE. http://www.invo.org.uk/. (accessed July 27, 2010).

13. Makela M, Stein K. Mind sharpeners for scientists. Int J Technol Assess Health Care. 2011;27:99-100.

14. McKenzie A, Hanley B. Consumer and community participation in health and medical research. Perth, Western Australia: University of Western Australia; 2007.

15. Mockford C, Staniszewska S, Griffiths F, et al. The impact of patient and public involvement on UK NHS healthcare. International Journal of Quality in Healthcare. In press.

16. Moher D, Hopewell S, Schultz K. Consort 2010 explanation and elaboration: Updated guidelines for reporting parallel group randomised trials. BMJ. 2010;340:c723.

17. Moher D, Schulz KF, Altman DG. The CONSORT statement: Revised recommendations for improving the quality of reports of parallel-group randomised trials. Lancet. 2001;357:11911194.

18. National Institutes of Health [WWW document]. URL http://copr.nih.gov/ (accessed April 1, 2011). 
19. Research Design Services. http://www.nihr.ac.uk/ infrastructure/Pages/infrastructure_research_design_services. aspx (accessed July 27, 2010).

20. Shea B, Santesso N, Qualman A, et al. Cochrane Musculoskeletal Consumer Group. Consumer-driven health care: Building partnerships in research. Health Expect. 2005;8/4:352-359.

21. Staley K. Exploring impact: Public involvement in NHS, public health and social care research. Eastleigh: INVOLVE; 2009.

22. Staniszewska S, et al. Developing the evidence base of patient and public involvement in health and social care research: The case for measuring impact. Int J Consum Stud. 2011. in press.
23. Streiner DL, Norman GR. Health measurement scales. A practical guide to their development and use. Oxford: Oxford Medical Publications; 2005.

24. The National Health and Medical Research Council. http://www.nhmrc.gov.au/_files_nhmrc/file/service (accessed April 1, 2011).

25. Ware JE, Sherbourne CD. The MOS 36-Item Short-Form Health Survey (SF-36@): I. conceptual framework and item selection. Med Care. 1992;30:473-483.

26. Wright D, Foster C, Amir Z, Elliott J, Wilson R. Critical appraisal guidelines for assessing the quality and impact of user involvement in research. Health Expect. 2010;13:359-368. 